"Mircea cel Batran" Naval Academy Scientific Bulletin, Volume XIX - 2016 - Issue 2

The journal is indexed in: PROQUEST / DOAJ / Crossref / EBSCOhost / INDEX COPERNICUS / DRJI / OAJI I JOURNAL INDEX I I2OR / SCIENCE LIBRARY INDEX / Google Scholar / Academic Keys/ ROAD Open Access I Academic Resources / Scientific Indexing Services / SCIPIO / JIFACTOR

\title{
ALGORITHM FOR DETERMINING THE MAXIMUM VALUE OF ALL SUBGRAPHS WITH $k$ VERTICES
}

\author{
Paul VASILIU ${ }^{1}$ \\ ${ }^{1}$ Lecturer Ph.D. Eng. Department of Electrical Engineering and Electronics, „Mircea cel Batran” Naval \\ Academy
}

Abstract:In this paper we will prove how all subgraphs with $k$ vertices and weighted edges of a graph can be generated and how can be computed the maximum value of all subgraphs with $k$ vertices."The paper will include a written $\mathrm{C}++$ program that implements the presented algorithm. Moreover, a use case scenario for this algorithm will be described"[1].

Keywords: graph, subgraph, compute, algorithm, program

\section{INTRODUCTION}

"Let it be $X=\left\{x_{1}, x_{2}, \cdots, x_{n}\right\}$ the set of vertices; we have the application $\Gamma: X \rightarrow P(X)$ and the graph $G=(X, \Gamma)$. Let it be $A=\left(a_{i j}\right)_{\substack{i=1,2, m \\ j=1,2, n}}$ with the elements $a_{i j}=\left\{\begin{array}{lll}1 & \text { if } & x_{j} \in \Gamma\left(x_{i}\right) \\ 0 & \text { if } & x_{j} \notin \Gamma\left(x_{i}\right)\end{array}\right.$ the adjacency matrix of graph $G=(X, \Gamma)$ “ $[1]$.

Let it be $U=\left\{\left(x_{i}, x_{j}\right) \mid x_{j} \in \Gamma\left(x_{i}\right)\right\}$ the set of edges of the graph. Let us assume that the graph has weighted edges $l\left(x_{i}, x_{j}\right)$ with values greater than 0 for every $\left(x_{i}, x_{j}\right) \in U$.

We define as value for the graph $G=(X, \Gamma)$, the number $l(G)=\Sigma_{\left(x_{i}, x_{j}\right) \in U} l\left(x_{i}, x_{j}\right)$.

Let it $\quad$ be $X^{p}=\left\{x_{i_{1}}, x_{i_{2}}, \cdots, x_{i_{k}}\right\} \subset\left\{x_{1}, x_{2}, \cdots, x_{n}\right\}$ the subset, not null, of vertices of set $X$.

Let it be $\Gamma^{s}: X^{t} \rightarrow P\left(X^{v}\right)$ the application defined $\Gamma^{g}\left(x_{i_{p}}\right)=\Gamma\left(x_{i_{p}}\right) \cap X^{s}$, by for every $x_{i_{p}} \in X^{t}$. Pair $G^{r}=\left(X^{r}{ }_{0} \Gamma^{r}\right)$ is called the subgraph of the graph $G=(X, \Gamma)$ defined by the set of vertices $X^{t}$.

Let it be $U^{r}=\left\{\left(x_{i}, x_{j}\right) \mid x_{j} \in \Gamma^{r}\left(x_{i}\right)\right\}$ the set of edges of the subgraph $G^{r}=\left(X^{t}, \Gamma^{r}\right)$.

Let it be defined the number $\left(G^{\prime}\right)=\sum_{\left(x_{i}, x_{j}\right) \in U^{\prime}} l\left(x_{i}, x_{j}\right)$ for the subgraph $G=(X, \Gamma)$.

"Inthis paper, we will prove how can be generated all the subgraphs of $k$ vertices of a given graph and how their values can be computed" [1].

We will also determine the maximum value of all subgraphswith $k$ vertices.

We will present a program written in $\mathrm{C}++$; that receives as input a graph and the number $\mathrm{k}$, representing the number of vertices. The program generates all the subgraphs defined by $k$ vertices and their values, and then determine the maximum value of all subgraphs.

The paper includes a usage example for the program. In the end, we will present the future work for this research.

\section{ALGORITHM}

In this section, we will show how can be generated all the subgraphs defined by $k$ vertices of a given graph and also, how the values for the subgraphs are computed.

"For this purpose, we generated through backtracking all the subsets of $k$ distinct elements of the set $\{1,2, \cdots, n\}$ in which the order does not matter. In other words, we generated all the combinations of kelements of set $\{1,2, \cdots, n\}$. All these subsets are saved in a file named sg.txt. The elements of each subset $\left\{i_{1}, i_{2}, \cdots, i_{k}\right\}$ represent the indexes of the subgraph vertices that will be generated and which value will be computed. The value of the subgraph $G^{r}=\left(X^{t}{ }_{0} \Gamma^{r}\right)$ defined by the vertices $x_{i_{1}}, x_{i_{2}}, \cdots, x_{i_{k}}$ equals to $l=\sum_{\left(x_{i} x_{j}\right) \in U}, l\left(x_{i}, x_{j}\right)$ " [1].

"Subsets are extracted successively from file sg.txt. For each subset $\left\{i_{1}, i_{2}, \cdots, i_{k}\right\}$ has generated the graph defined by vertices $x_{i_{1}}, x_{i_{2}},{ }, \ldots, x_{i_{k}}$ and computed its value.

When generating each subgraph, we take into consideration the adjacency matrix $A^{s}$ of the subgraph $G^{b}=\left(X^{s}, \Gamma^{b}\right)$, can be obtained from the adjacency matrix Aof graph $G=(X, \Gamma)$ keeping the rows and columns with indexesi $i_{1}, i_{2}, \cdots, i_{k}$ and canceling all the rows and columns from matrix $A$ with indexes $i \notin\left\{i_{1}, i_{2}, \cdots, i_{k}\right\}$ " [1].

Sgval.txt text file have stored all subgraphs and their values.It then reads this file and determines the maximum value of all subgraphs. 
"Mircea cel Batran" Naval Academy Scientific Bulletin, Volume XIX - 2016 - Issue 2

The journal is indexed in: PROQUEST / DOAJ / Crossref / EBSCOhost / INDEX COPERNICUS / DRJI / OAJI I JOURNAL INDEX I I2OR / SCIENCE LIBRARY INDEX / Google Scholar / Academic Keys/ ROAD Open Access I Academic Resources / Scientific Indexing Services / SCIPIO / JIFACTOR

\section{IMPLEMENTATION}

Below we present the program written in $\mathrm{C}++$ that implements the described algorithm.

The program receives as input the text file associated with the graph $G=(x, \Gamma)$, the number of vertices and the vertices $x_{i_{1}}, x_{i_{2}},{ }^{n}, x_{i_{k}}$ of the subgraph that will be generated.

"The program outputs the number of vertices, the number of edges, the adjacency matrix, the edges of graph $G=(X, \Gamma)$ and all subgraphs of a maximum value" [1]

For the subgraph $G^{s}=\left(X^{b}, \Gamma^{s}\right)$ we display the adjacency matrix, the edges of the subgraph and value respectively subgraph.

\#include <stdio.h>

\#include <conio.h $>$

\#include <malloc.h>

\#define dim 1000

int va[100];

typedef struct arc

\{

int $\mathrm{x}$; int $\mathrm{y}$; int val;

\}edge;

edge mu[dim],musg[dim]; // mu array of edges

// Matrix allocation

int ${ }^{* *}$ alocmat (int $\mathrm{n}$ )

\{

int $\mathrm{i}$;

int ${ }^{* *} p=\left(\right.$ int $\left.{ }^{* *}\right)$ malloc $\left((n+1)^{\star}\right.$ sizeof (int *));

if $(p !=$ NULL)

for $(\mathrm{i}=0 ; \mathrm{i}<=\mathrm{n} ; \mathrm{i}++)$

$\mathrm{p}[\mathrm{i}]=\left(\right.$ int $\left.{ }^{*}\right)$ calloc $((\mathrm{n}+1)$, sizeof $(\mathrm{int}))$;

return $\mathrm{p}$;

\}

// Function for allocating the array

int *alocv(int n)

\{

int *p;

$\mathrm{p}=\left(\right.$ int $\left.{ }^{*}\right) \mathrm{malloc}\left(\mathrm{n}^{*}\right.$ sizeof(int));

return $\mathrm{p}$;

\}

// Function for displaying the array

void displayv(int $n$,int *v) //

\{

int i;

printf(" \{ ");

for $(\mathrm{i}=0 ; \mathrm{i}<\mathrm{n}-1 ; \mathrm{i}++)$

printf(" \%d , ",v[i]);

printf(" \%d ",v[i]);

printf(" \} $\ln ")$;

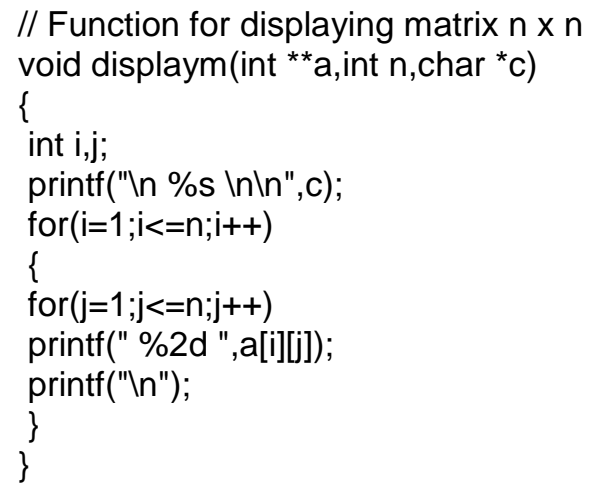

// Function for displaying matrix $\mathrm{n} \times \mathrm{n}$ void displaym(int ${ }^{* *} a$,int $n$, char ${ }^{*} \mathrm{c}$ )

\{

int $\mathrm{i}, \mathrm{j}$;

printf("In \%s Inln",c);

for $(i=1 ; i<=n ; i++)$

\{

for $(j=1 ; j<=n ; j++)$

printf(" \%2d ",a[i][j]);

printf("In");

\}

\}

// Function for displaying the subgraph

void displaysg(char ${ }^{*} \mathrm{c}$,int $\mathrm{k}$,int ${ }^{*} \mathrm{v}$ )

\{

int $\mathrm{i}, \mathrm{j}$;

printf("Inln \%s ",c);

displayv $(\mathrm{k}, \mathrm{v})$;

printf("Inln");

\}

// Function for displaying matrix $\mathrm{n} \times \mathrm{n}$

void displaymm(int ${ }^{* \star a}$, int $n$,char ${ }^{*} \mathrm{c}$,int $\mathrm{k}$,int ${ }^{*} \mathrm{v}$ )

\{

int $\mathrm{i}, \mathrm{j}$;

printf("In \%s ",c);

$\operatorname{displayv}(\mathrm{k}, \mathrm{v})$;

for $(\mathrm{i}=1 ; \mathrm{i}<=\mathrm{n} ; \mathrm{i}++)$

\{

for $(j=1 ; j<=n ; j++)$

printf(" \%2d ",a[i][j]);

printf("In");

\}

// Function for searching $\mathrm{k}$ in $\mathrm{v}$

int search(int k,int n,int *v)

\{

int i,found;

found $=0$;

for $(\mathrm{i}=0 ; \mathrm{i}<\mathrm{n} ; \mathrm{i}++)$

if $(\mathrm{v}[\mathrm{i}]==\mathrm{k})$

\{

found=1;

break;

\}

return found;

\}

//Function for reading $\mathrm{n}, \mathrm{m}$

int read_n_m(int \&n, int \&m, char *name)

\{

FILE *f;

if((f=fopen(name,"r"))!= NULL)

\{

fscanf(f,"\%d \%d",\&n,\&m); 
"Mircea cel Batran" Naval Academy Scientific Bulletin, Volume XIX - 2016 - Issue 2

The journal is indexed in: PROQUEST / DOAJ / Crossref / EBSCOhost / INDEX COPERNICUS / DRJI / OAJI I JOURNAL INDEX I I2OR / SCIENCE LIBRARY INDEX / Google Scholar / Academic Keys/ ROAD Open Access I Academic Resources / Scientific Indexing Services / SCIPIO / JIFACTOR

fclose(f);

return 1;

\}

else

return 0;

\}

// Function for displaying the edges of the graph void display_edges(int m)

\{

int i,val=0;

printf("In Graph G has \%d edges In $\backslash n ", m)$;

for $(i=1 ; i<=m ; i++)$

\{

printf(" Edge \%d:It ( x\%d , x\%d ) with value \%d

In",i,mu[i].x,mu[i].y,mu[i].val);

val+=mu[i].val;

\}

printf(" The value of the graph is \%d $\mid n ", v a l)$; printf("In");

//Function for reading the input file and

//building the adjacency matrix

int read_graph(char *name, int **a)

\{

int i,j,x,y,val,n,m;

FILE *f;

if((f=fopen(name,"r")) != NULL)

\{

fscanf(f,"\%d \%d",\&n,\&m);

for $(i=1 ; i<=m ; i++)$

\{

fscanf(f," \%d

$\% d ", \& m u[i] . x, \& m u[i] . y, \& m u[i] . v a l) ;$

$\mathrm{a}[\mathrm{mu}[\mathrm{i}] \cdot \mathrm{x}][\mathrm{mu}[\mathrm{i}] \cdot \mathrm{y}]=1$;

\}

fclose(f);

return 1;

\}

else

return 0;

\}

//Function for generating

//the subgraph adjacency matrix

void gen(int $n$,int ${ }^{* *}$ a, int $k$,int ${ }^{*} v$,int **ap)

\{

int $\mathrm{i}, \mathrm{j}$;

for $(\mathrm{i}=1 ; \mathrm{i}<=\mathrm{n} ; \mathrm{i}++)$

if $(\operatorname{search}(\mathrm{i}, \mathrm{k}, \mathrm{v})==1)$

for $(j=1 ; j<=n ; j++)$

if $(\operatorname{search}(j, k, v)==1)$

ap[i][j]=a[i][i];

\}

// Function for displaying the edges of the subgraph
// Compute the value of the subgraph int display_edges_s(int $n$,int $m$,int **ap,int *vval,int nsg)

\{

int $\mathrm{i}, \mathrm{j}, \mathrm{p}, \mathrm{val}=0$;

for $(p=1, i=1 ; i<=n ; i++)$

for $(j=1 ; j<=n ; j++)$

if $(\operatorname{ap}[i][j]==1)$

\{

musg[p].x=i;

musg[p].y=j;

$\mathrm{p}++$;

\}

p--;

for $(i=1 ; i<=p ; i++)$

for $(j=1 ; j<=m ; j++)$

if(musg[i].x==mu[j].x \&\& musg[i].y==mu[j].y)

musg[i].val=mu[j].val;

printf("In The subgraph has \%d edges In\n",p); for $(i=1 ; i<=p ; i++)$

\{

printf(" The edge \%d:It ( $x \% d, x \% d$ ) of value $\% d$

In",i,musg[i].x,musg[i].y,musg[i].val);

val+=musg[i].val;

\}

printf("In");

vval[nsg-1]=val;

return val;

\}

"[1]

//Function for reading the vertices of the next subgraph

void readf(FILE *f,int $n$,int * $v$ )

\{

int $i$;

for $(i=0 ; i<n ; i++)$

fscanf(f," \%d ",\&v[i]);

\}

//Matrix initialization

void initm(int $n$,int **a)

\{

int i,j;

for $(\mathrm{i}=1 ; \mathrm{i}<=\mathrm{n} ; \mathrm{i}++)$

for $(j=1 ; j<=n ; j++)$

$\mathrm{a}[\mathrm{i}][\mathrm{j}]=0$;

\}

//Function for initializing array $x$

void init(int *x,int k)

\{

$x[k]=0$;

\}

//Function for testing the solution

int sol(int $k$,int $p)$ 
"Mircea cel Batran" Naval Academy Scientific Bulletin, Volume XIX - 2016 - Issue 2

The journal is indexed in: PROQUEST / DOAJ / Crossref / EBSCOhost / INDEX COPERNICUS / DRJI / OAJI I JOURNAL INDEX I I2OR / SCIENCE LIBRARY INDEX / Google Scholar / Academic Keys/ ROAD Open Access I Academic Resources / Scientific Indexing Services / SCIPIO / JIFACTOR

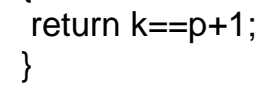

// Function for compute the value of $n$ ! int fact(int $n$ )

\{

if $(n==0)$

return 1;

else
// Function for compute the value of cnk int comb(int $n$,int k)

\{ \} return fact(n)/(fact(k)*fact(n-k));

// Function for compute the maximum value int $\max ($ int $n$,int * $v$ )

\{

int i,max;

$\max =\mathrm{v}[0] ;$

for $(\mathrm{i}=1 ; \mathrm{i}<\mathrm{n} ; \mathrm{i}++)$

if(v[i]>max)

$\max =\mathrm{v}[\mathrm{i}]$;

\}

return max;

//Function for reading the sgval.txt file void readfsgmax(int valmax,int k,int nsg)

\{

FILE *f;

int $\mathrm{i}, \mathrm{j},{ }^{*} \mathrm{v}, \mathrm{val}$;

$\mathrm{f}=$ fopen("sgval.txt","r");

$\mathrm{v}=\mathrm{alocv}(\mathrm{k})$;

for $(\mathrm{j}=0 ; j<\mathrm{nsg} ; \mathrm{j}++)$

\{

for $(i=0 ; i<k ; i++)$

fscanf(f,"\%d ",\&v[i]);

fscanf(f,"\%d",\&val);

if(val==valmax)

\{

printf(" The subgraph \{ ");

for $(i=0 ; i<k ; i++)$

printf(" \%d ",v[i]);

printf("\} has maximum value \%d In",valmax);

\}

\}

fclose(f);

\}

// The main function

int main()

\{

int

$\mathrm{n}, \mathrm{m}, \mathrm{k},{ }^{*} \mathrm{vval},{ }^{* *} \mathrm{a},{ }^{* *} \mathrm{ap},{ }^{*} \mathrm{v}, \mathrm{nsg}=0, \mathrm{i}, \mathrm{x}[100]$, valsg,valma $\mathrm{x}$;

char name[30];

FILE *f, *fval;

printf("In File name : ");

fflush(stdin);

gets(name);

if(read_n_m(n,m,name))

\{

printf("In Number of vertices \%d In",n);

printf(" Number of edges \%d In",m); 
"Mircea cel Batran" Naval Academy Scientific Bulletin, Volume XIX - 2016 - Issue 2

The journal is indexed in: PROQUEST / DOAJ / Crossref / EBSCOhost / INDEX COPERNICUS / DRJI / OAJI / JOURNAL INDEX I I2OR / SCIENCE LIBRARY INDEX / Google Scholar / Academic Keys/ ROAD Open Access I Academic Resources / Scientific Indexing Services / SCIPIO / JIFACTOR

getch () ;

$\mathrm{a}=$ alocmat(n);

if(read_graph(name,a))

\{

displaym(a,n," Graph adjacency matrix $\backslash n ") ;$

display_edges $(\mathrm{m})$;

printf(" The number of vertices of the subgraph = ");

scanf("\%d",\&k);

"[1].

if $(1<=\mathrm{k} \& \& \mathrm{k}<=\mathrm{n})$

\{

vval=alocv $(\operatorname{comb}(\mathrm{n}, \mathrm{k}))$;

$\mathrm{f}=$ fopen("sg.txt","w");

for $(i=1 ; i<=n ; v a[i]=i, i++)$;

$\operatorname{back}(\mathrm{x}, 1, \mathrm{n}, \mathrm{k}, \mathrm{f})$;

fclose $(\mathrm{f})$;

$\mathrm{v}=\operatorname{alocv}(\mathrm{k})$;

ap=alocmat(n);

f=fopen("sg.txt","r");

fval=fopen("sgval.txt", "w");

while(!feof(f))

\{

readf(f,k,v);

nsg++;

gen(n,a,k,v,ap);

displaysg(" Subgraph ",k,v);

displaymm(ap,n," Subgraph adjacency matrix

",k,v);

valsg=display_edges_s(n,m,ap,vval,nsg);

printf(" The value of the subgraph is \%d

In",valsg);

for $(i=0 ; i<k ; i++)$

fprintf(fval,"\%d ",v[i]);

fprintf(fval,"\%dln",valsg);

initm(n,ap);

getch();

\}

"[1].

fclose $(f)$;

fclose(fval);

printf("In\n Number of subgraphs with \%d vertices = \%d Inlnln",k,nsg);

valmax=max(nsg,vval);

printf(" The maximum value is \%d In In",valmax);

getch();

readfsgmax(valmax,k,nsg);

\}

else

printf(" Undefinied problem In");

\}

getch();

\section{AN EXAMPLE}

Let us consider the directed graph $G=\left(X_{v} \Gamma\right)$, with weighted edges, definied by the set of vertices $X=\left\{x_{1}, x_{2}, x_{\mathrm{a}}, x_{4}, x_{5}\right\}$ and the application $\Gamma: X \rightarrow P(X) \quad$ definied by the equalities: $\Gamma\left(x_{1}\right)=\left\{x_{1}, x_{3}, x_{5}\right\}, \quad \Gamma\left(x_{2}\right)=\left\{x_{1}, x_{4}, x_{5}\right\}$, $\Gamma\left(x_{\mathrm{a}}\right)=\left\{x_{2}, x_{4}, x_{5}\right\}$, $\Gamma\left(x_{4}\right)=\left\{x_{2}, x_{2}\right\}$, $\Gamma\left(x_{5}\right)=\left\{x_{1}, x_{9}, x_{4}\right\}$.

Edges values are given in Table 1:

\begin{tabular}{|c|c|c|c|}
\hline Edge & Value & Edge & Value \\
\hline$\left(x_{1}, x_{1}\right)$ & 3 & $\left(x_{3}, x_{4}\right)$ & 17 \\
\hline$\left(x_{1}, x_{2}\right)$ & 3 & $\left(x_{2}, x_{5}\right)$ & 1 \\
\hline$\left(x_{1}, x_{5}\right)$ & 2 & $\left(x_{4}, x_{2}\right)$ & 4 \\
\hline$\left(x_{2}, x_{1}\right)$ & 5 & $\left(x_{4}, x_{2}\right)$ & 6 \\
\hline$\left(x_{2}, x_{4}\right)$ & 3 & $\left(x_{5}, x_{1}\right)$ & 2 \\
\hline$\left(x_{2}, x_{5}\right)$ & 6 & $\left(x_{5}, x_{2}\right)$ & 1 \\
\hline$\left(x_{2}, x_{2}\right)$ & 4 & $\left(x_{5}, x_{4}\right)$ & 9 \\
\hline
\end{tabular}

Table 1Edges values

The adjacency matrix of the graph $G=\left(X_{*} \Gamma\right)$ is:

$A=\left(\begin{array}{lllll}1 & 0 & 1 & 0 & 1 \\ 1 & 0 & 0 & 1 & 1 \\ 0 & 1 & 0 & 1 & 1 \\ 0 & 1 & 1 & 0 & 0 \\ 1 & 0 & 1 & 1 & 0\end{array}\right)$.

"The program read as input a text file with name graph.txt The file has on the first line the number $\mathrm{n}$, representing the number of vertices, and the number $\mathrm{m}$, representing the number of edges separated by one blank space. On the next $\mathrm{m}$ lines, we find the edges of the subgraphand their values. For each graph from our example, the input file graph.txt is the following:" [1].

514

113

133

152

215

243

256

324

3417

351

424

436

512

531

549

For $k=3$, file sg.txt is: 
"Mircea cel Batran" Naval Academy Scientific Bulletin, Volume XIX - 2016 - Issue 2 The journal is indexed in: PROQUEST / DOAJ / Crossref / EBSCOhost / INDEX COPERNICUS / DRJI / OAJI / JOURNAL INDEX I I2OR / SCIENCE LIBRARY INDEX / Google Scholar / Academic Keys/ ROAD Open Access I Academic Resources / Scientific Indexing Services / SCIPIO / JIFACTOR

123

124

125

134

135

145

234

235

245

345

For $k=3$, file sgval.txt is:

12315

12415

12518

13429

13512

14516

23434

23512

24522

34534

"A run execution example of the program is:

File name : graph.txt

Number of vertices 5

Number of edges 14

Graph adjacency matrix

$\begin{array}{lllll}1 & 0 & 1 & 0 & 1 \\ 1 & 0 & 0 & 1 & 1 \\ 0 & 1 & 0 & 1 & 1 \\ 0 & 1 & 1 & 0 & 0 \\ 1 & 0 & 1 & 1 & 0\end{array}$

Graph G has 14 edges

Edge 1: $\quad(x 1, x 1)$ with value 3

Edge 2: $\quad(x 1, x 3)$ with value 3"[1].

Edge 3: $\quad(x 1, x 5)$ with value 2

Edge 4: $\quad(x 2, x 1)$ with value 5

Edge 5: $\quad(x 2, x 4)$ with value 3

Edge 6: $\quad(x 2, x 5)$ with value 6

Edge 7: $\quad(x 3, x 2)$ with value 4

Edge 8: $\quad(x 3, x 4)$ with value 17

Edge 9: $\quad(x 3, x 5)$ with value 1

Edge 10: $\quad(x 4, x 2)$ with value 4

Edge 11: $\quad(x 4, x 3)$ with value 6

Edge 12: $\quad(x 5, x 1)$ with value 2

Edge 13: $\quad(x 5, x 3)$ with value 1

Edge 14: $\quad(x 5, x 4)$ with value 9

The value of the graph is 66

The number of vertices of the subgraph $=3$

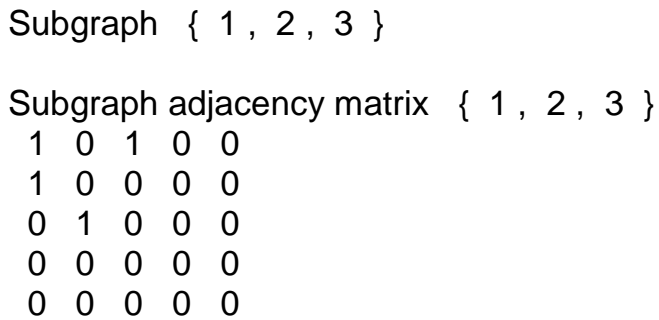

The subgraph has 4 edges

The edge 1: $\quad(x 1, x 1)$ of value 3

The edge 2: $\quad(x 1, x 3)$ of value 3

The edge 3: $\quad(x 2, x 1)$ of value 5

The edge 4: $\quad(x 3, x 2)$ of value 4

The value of the subgraph is 15

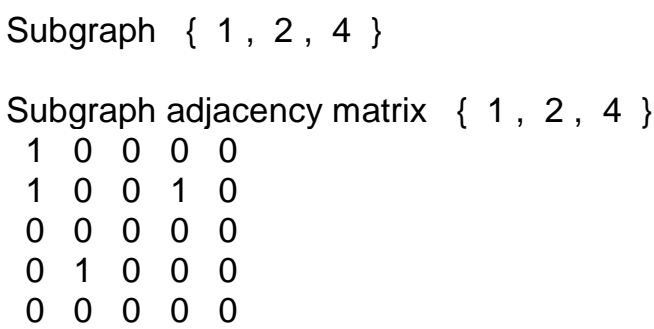

The subgraph has 4 edges

The edge 1: $\quad(x 1, x 1)$ of value 3

The edge 2: $\quad(x 2, x 1)$ of value 5

The edge 3: $\quad(x 2, x 4)$ of value 3

The edge 4: $\quad(x 4, x 2)$ of value 4

The value of the subgraph is 15

Subgraph $\{1$
$\begin{array}{ccccc}\text { Subgraph adjace } \\ 1 & 0 & 0 & 0 & 1 \\ 1 & 0 & 0 & 0 & 1 \\ 0 & 0 & 0 & 0 & 0 \\ 0 & 0 & 0 & 0 & 0 \\ 1 & 0 & 0 & 0 & 0\end{array}$

The subgraph has 5 edges

The edge 1: $(x 1, x 1)$ of value 3

The edge 2: $\quad(x 1, x 5)$ of value 2

The edge 3: $\quad(x 2, x 1)$ of value 5

The edge 4: $\quad(x 2, x 5)$ of value 6

The edge 5: $\quad(x 5, x 1)$ of value 2

The value of the subgraph is 18

Subgraph $\{1,3,4\}$ 
"Mircea cel Batran" Naval Academy Scientific Bulletin, Volume XIX - 2016 - Issue 2 The journal is indexed in: PROQUEST / DOAJ / Crossref / EBSCOhost / INDEX COPERNICUS / DRJI / OAJI / JOURNAL INDEX I I2OR / SCIENCE LIBRARY INDEX / Google Scholar / Academic Keys/ ROAD Open Access I Academic Resources / Scientific Indexing Services / SCIPIO / JIFACTOR

\begin{tabular}{ccccc}
\multicolumn{8}{c}{ Subgraph adjace } \\
1 & 0 & 1 & 0 & 0 \\
0 & 0 & 0 & 0 & 0 \\
0 & 0 & 0 & 1 & 0 \\
0 & 0 & 1 & 0 & 0 \\
0 & 0 & 0 & 0 & 0
\end{tabular}

The subgraph has 4 edges

The edge 1: $\quad(x 1, x 1)$ of value 3

The edge 2: $\quad(x 1, x 3)$ of value 3

The edge 3: $\quad(x 3, x 4)$ of value 17

The edge 4: $\quad(x 4, x 3)$ of value 6

The value of the subgraph is 29

Subgraph $\{1,3,5\}$

Subgraph adjacency matrix $\{1,3,5\}$

$\begin{array}{lllll}1 & 0 & 1 & 0 & 1 \\ 0 & 0 & 0 & 0 & 0 \\ 0 & 0 & 0 & 0 & 1 \\ 0 & 0 & 0 & 0 & 0 \\ 1 & 0 & 1 & 0 & 0\end{array}$

"The subgraph has 6 edges

The edge 1: $\quad(x 1, x 1)$ of value 3

The edge 2: $\quad(x 1, x 3)$ of value $3 "[1]$.

The edge 3: $\quad(x 1, x 5)$ of value 2

The edge 4: $\quad(x 3, x 5)$ of value 1

The edge 5: $\quad(x 5, x 1)$ of value 2

The edge $6: \quad(x 5, x 3)$ of value 1

The value of the subgraph is 12

Subgraph $\{1,4,5\}$

Subgraph adjacency matrix $\{1,4,5\}$

$\begin{array}{lllll}1 & 0 & 0 & 0 & 1\end{array}$

$\begin{array}{lllll}0 & 0 & 0 & 0 & 0\end{array}$

$\begin{array}{lllll}0 & 0 & 0 & 0 & 0\end{array}$

$0 \begin{array}{lllll}0 & 0 & 0 & 0 & 0\end{array}$

$\begin{array}{lllll}1 & 0 & 0 & 1 & 0\end{array}$

The subgraph has 4 edges

The edge 1: $\quad(x 1, x 1)$ of value 3

The edge 2: $\quad(x 1, x 5)$ of value 2

The edge 3: $\quad(x 5, x 1)$ of value 2

The edge 4: $\quad(x 5, x 4)$ of value 9

The value of the subgraph is 16

Subgraph $\{2,3,4\}$

Subgraph adjacency matrix $\{2,3,4\}$

$\begin{array}{lllll}0 & 0 & 0 & 0 & 0\end{array}$

$$
\begin{array}{lllll}
0 & 0 & 0 & 1 & 0 \\
0 & 1 & 0 & 1 & 0 \\
0 & 1 & 1 & 0 & 0 \\
0 & 0 & 0 & 0 & 0
\end{array}
$$

"The subgraph has 5 edges

The edge 1: $\quad(x 2, x 4)$ of value 3

The edge 2: $\quad(x 3, x 2)$ of value 4 "'[1].

The edge 3: $\quad(x 3, x 4)$ of value 17

The edge 4: $\quad(x 4, x 2)$ of value 4

The edge 5: $\quad(x 4, x 3)$ of value 6

The value of the subgraph is 34

Subgraph $\{2,3,5\}$

Subgraph adjacency matrix $\{2,3,5\}$

$$
\begin{array}{lllll}
0 & 0 & 0 & 0 & 0 \\
0 & 0 & 0 & 0 & 1 \\
0 & 1 & 0 & 0 & 1 \\
0 & 0 & 0 & 0 & 0 \\
0 & 0 & 1 & 0 & 0
\end{array}
$$

"The subgraph has 4 edges

The edge 1: $\quad(x 2, x 5)$ of value 6

The edge 2: $\quad(x 3, x 2)$ of value 4

The edge 3: $\quad(x 3, x 5)$ of value 1

The edge 4: $\quad(x 5, x 3)$ of value 1

The value of the subgraph is 12

Subgraph $\{2,4,5\}$

Subgraph adjacency matrix $\{2,4,5\}$

$\begin{array}{lllll}0 & 0 & 0 & 0 & 0 \\ 0 & 0 & 0 & 1 & 1 \\ 0 & 0 & 0 & 0 & 0 \\ 0 & 1 & 0 & 0 & 0 \\ 0 & 0 & 0 & 1 & 0\end{array}$

The subgraph has 4 edges

The edge 1: $\quad(x 2, x 4)$ of value 3

The edge 2: $\quad(x 2, x 5)$ of value 6

The edge 3: $\quad(x 4, x 2)$ of value 4

The edge 4: $\quad(x 5, x 4)$ of value 9

The value of the subgraph is 22

Subgraph $\{3,4,5\}$

Subgraph adjacency matrix $\{3,4,5\}$

$\begin{array}{lllll}0 & 0 & 0 & 0 & 0 \\ 0 & 0 & 0 & 0 & 0 \\ 0 & 0 & 0 & 1 & 1\end{array}$ 
"Mircea cel Batran" Naval Academy Scientific Bulletin, Volume XIX - 2016 - Issue 2 The journal is indexed in: PROQUEST / DOAJ / Crossref / EBSCOhost / INDEX COPERNICUS / DRJI / OAJI I JOURNAL INDEX / I2OR / SCIENCE LIBRARY INDEX / Google Scholar / Academic Keys/ ROAD Open Access I Academic Resources / Scientific Indexing Services / SCIPIO / JIFACTOR

$\begin{array}{lllll}0 & 0 & 1 & 0 & 0\end{array}$

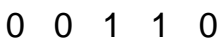

The subgraph has 5 edges

The edge 1: $\quad(x 3, x 4)$ of value 17

The edge 2: $\quad(x 3, x 5)$ of value 1

The edge 3: $(x 4, x 3)$ of value 6

The edge 4: $\quad(x 5, x 3)$ of value 1

The edge 5: $\quad(x 5, x 4)$ of value 9

The value of the subgraph is 34

Number of subgraphs with 3 vertices $=10$

The maximum value is 34

\section{CONCLUSIONS}

"In this paper, we have presented an algorithm and a program written in C++ for generating all the subgraphs defined by $k$ gave vertices of a given graph with weighted edges" [1]. Furthermore, we computed the values of these subgraphs and the maximum value of all subgraphs with $k$ vertices.

From the large spectrum of future work we will enumerate:

determining the number of vertices of the subgraphs maximum value; determining the number of vertices of the subgraphs minimumvalue.

\section{BIBLIOGRAPHY}

[1] Vasiliu P., T. Pazara, Algorithm for generating allkvertices subgraphs and finding the values for each of the subgraphs, "Mircea cel Batran" Naval Academy Publishing House, Scientific Bulletin, Volume XVIII 2015 Issue nr.2, pg. 419-424, Constanta, Romania, ISSN 2392-8956, ISSN-L 1454-864X. 